\title{
Novel "Enhanced-Cognition" RFID Architectures on Organic/Paper Low-Cost Substrates Utilizing Inkjet Technologies
}

\author{
Li Yang, Amin Rida, Rushi Vyas, and Manos M. Tentzeris
}

Received 2 March 2007; Accepted 10 September 2007

Recommended by Junho Yeo

\begin{abstract}
The purpose of this paper is to present an overview of novel design and integration approaches for improved performance "enhanced-cognition" UHF passive and active radio frequency identification (RFID) tags. Antenna design rules are explained for a variety of applications. A strategy that is currently under development for embedding power sources and integration of sensors and integrated circuits (ICs) on low-cost organic substrates, such as liquid crystal polymer (LCP) and paper, enabling the use of inkjet-printing capability for the UHF frequency band, is discussed in the paper. The proposed technologies could potentially revolutionize RFID tags allowing for integrated sensing capabilities for various applications such as security, military, logistics, automotion, and pharmaceutics.
\end{abstract}

Copyright (c) 2007 Li Yang et al. This is an open access article distributed under the Creative Commons Attribution License, which permits unrestricted use, distribution, and reproduction in any medium, provided the original work is properly cited.

\section{INTRODUCTION}

Radio frequency identification (RFID) is a compact wireless technology which does not require line of sight (LoS) to communicate with the reader and allows for simultaneous $\mathrm{read} /$ write from multiple tags, as well as an easy remote and selective activation of sensor devices based on their unique IDs [1]. Due to the increasing demand for automatic identification, RFID could potentially find countless applications in different areas including retail level management, item level tracking, access control, animal tracking, vehicle security, and electronic toll collection [2].

At the same time and driven by several "cognitive-intelligence" applications [3] such as item-level tracking of temperature-sensitive products, pharmaceutical logistics, transport and storage of medical products or biosensing applications, a demand for inexpensive, low-power consumption, and durable wireless nodes with sensing capabilities has also increased tremendously. Compared with passive UHF RFID systems (maximum read range $\sim 10 \mathrm{~m}$ ), upon utilizing a power source, significant benefits in terms of read range can be achieved [4]. Furthermore, the hereby proposed potential ability to easily print RFID on organic substrates, such as paper, makes them an inexpensive candidate to create miniaturized wireless RFID/sensor modules at a significantly low cost [5].
In this paper, a brief outline of novel RFID architectures is presented for the purpose of optimized designs, enhancedrange and capabilities, easy fabrication, and low-cost. Material characterization, antenna design/matching guidelines, and sensors/power source integration are demonstrated, respectively.

\section{RFID ON ORGANIC SUBSTRATES}

There are three major considerations for the selection of the substrate material in RFID applications. First, the substrate should have conformal shape or flexibility in order to be placed on different shaped objects such as boxes, cylindrical bins, vehicles, and so forth. Secondly, since most RFID tags contain no extra packaging and are exposed directly to their surroundings, RFID tags must be able to withstand harsh industrial environments such as water vapor/humidity. The third requirement is to have a low dielectric constant value with low dielectric loss for optimal RF power efficiency and transmissivity in embedded configurations, as well as optimum power performance, especially in passive RFID systems, where the only power to the tag is the RF power from the reader in the vicinity.

Liquid crystal polymer (LCP) is a fairly new and promising thermoplastic organic material. It can be used as a lowcost dielectric material for high-volume, large-area processing methods that provide very reliable high-performance 


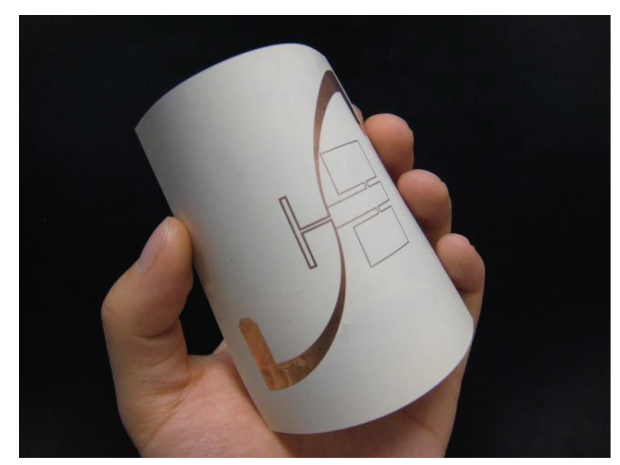

Figure 1: RFID tag on LCP.

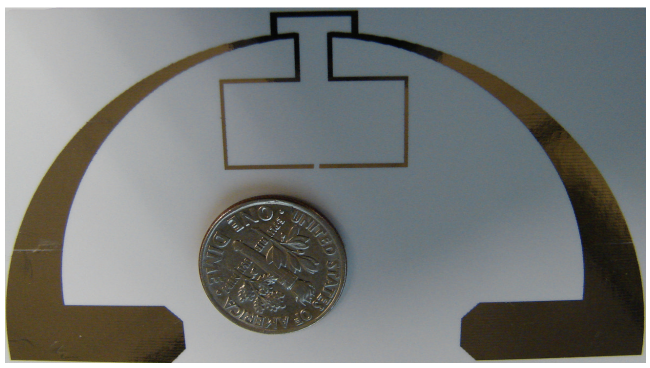

FIgURE 2: RFID tag on paper-based substrate.

circuits at low cost. It has low and stable water absorption rate around $0.04 \%$ [6], and a nearly constant dielectric constant of 3.1 over the entire RF range up to $110 \mathrm{GHz}$. In addition, LCP has a very low loss tangent of only 0.002 , which increases to only 0.0045 at $110 \mathrm{GHz}$, thereby making LCP very suitable in designing $\mathrm{mm}$-wave applications [7-9]. Low thermal expansion characteristics of LCP also make it ideal as a high frequency packaging material. The controllable coefficient of thermal expansion (CTE) of LCP can be engineered to match copper, silicon, or GaAs, thereby making metallization on it easier. Multilayer circuits in LCP are also possible due to two types of LCP material with different melting temperatures making it ideal for system on package designs. LCP is flexible, recyclable, impervious to most chemicals, and it is stable up to its high melting temperature making LCP an ideal choice for circuits operating in all kinds of environments. A typical passive RFID module is demonstrated in Figure 1. This antenna was fabricated on a $4 \mathrm{~mm}$ thick LCP substrate.

On the other side, paper is considered one of the best organic substrates candidates for RFID applications due to many reasons. Paper is not only environmentally friendly, but can also undergo large reel-to-reel processing. This makes paper the lowest cost material made for mass production and increased demand. Paper also has low surface profile with appropriate coating which is important for processes such as fast printing conductive paste inkjet printing. This process can be used instead of conventional metal etching techniques. In addition, paper is compatible with circuit printing by direct write methodologies [10]. This is one of its biggest advantages since active tags require additional mod-

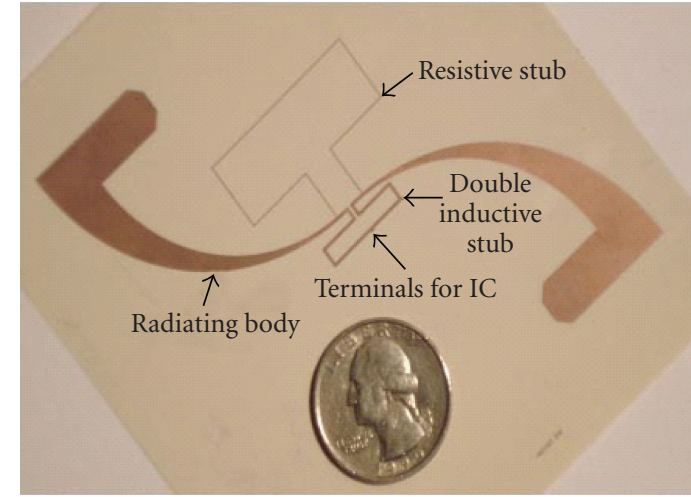

FIGURE 3: Typical RFID tag architecture.

ules like power sources and sensors to be mounted on or embedded in the substrate. A fast process like inkjet printing can be used efficiently to print these modules on or in the paper substrate. Paper also has the capability of hosting nanoscale additives to make the substrate immune to certain occurrences such as minor flames (i.e., fire-retardant textiles) [11]. Paper can also be made hydrophobic by adding the appropriate coating. Most importantly, its dielectric constant is close to air's [12] which means that electromagnetic power can penetrate easily even if the RFID is embedded inside substrates.

Electrical properties such as the dielectric constant and loss tangent can be effectively and accurately characterized up to $50 \mathrm{GHz}$ by using various methods such as microstrip ring resonator, cavity resonators, and parallel plate resonators [13]. Preliminary characterization results have showed that dielectric constant ranges from 1.6 to 3.2 depending on the thickness and process used (such as amount of hydrophobic coating used inside the paper substrate) and $\tan \delta\left(\sim 10^{-2}\right)$ for papers at UHF frequencies. A passive RFID module is demonstrated in Figure 2. An inkjet-printing technique is used in the antenna fabrication.

\section{NOVEL TECHNIQUES FOR UHF RFID ANTENNAS}

The UHF RFID bands range from $860 \mathrm{MHz}$ to $954 \mathrm{MHz}$ [14]. Different regions (such as North America, Europe, Australia) abide to different rules and regulations, such as frequency range $(866-868 \mathrm{MHz}$ in Europe and $902-928 \mathrm{MHz}$ in North America), power emission levels, number of channels used, and spurious limits. Moreover, the UHF frequency band allows for a much higher data rate over other bands typically used (e.g., the HF band operating at $13.56 \mathrm{MHz}$ ) and a higher read range $(\sim 30 \mathrm{ft}$ for passive tags and $\sim 100 \mathrm{ft}$ for semipassive or active tags).

A half wavelength antenna is typically used in RFID applications due to its omnidirectional radiation pattern hence enabling the tags' communication with the RFID reader in any orientation and for a variety of environments. A proposed topology of a typical passive UHF RFID is demonstrated in Figure 3. 


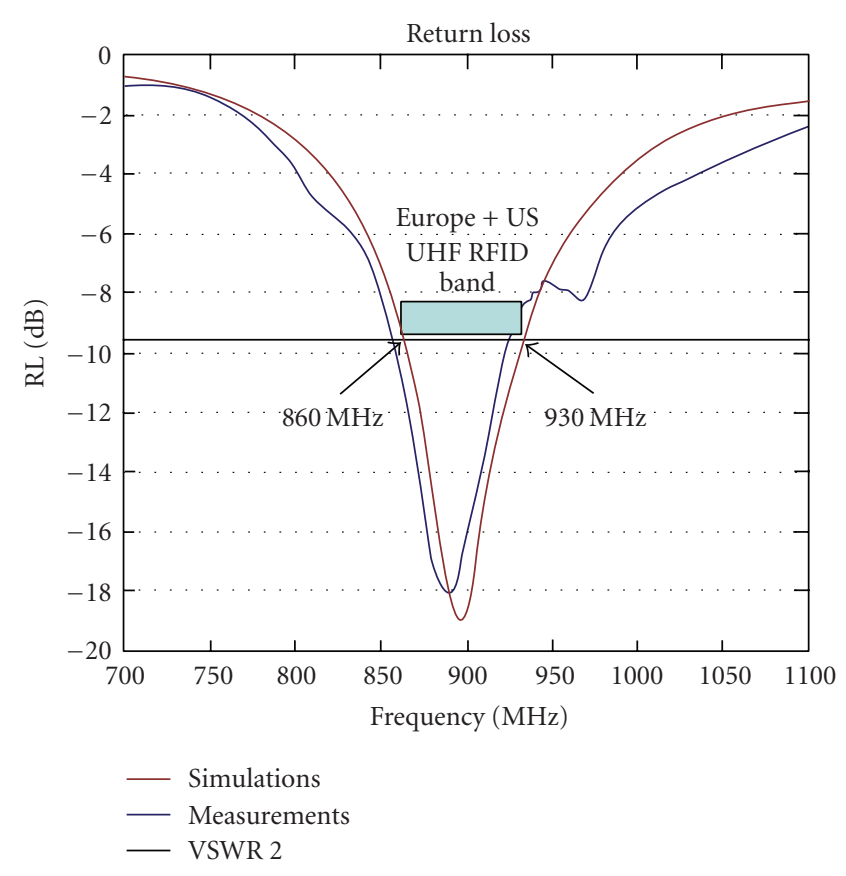

Figure 4: Return loss of the wideband RFID tag.

\subsection{Compact antennas with a universal/global bandwidth}

The dimensions of the antenna shown in Figure $3(7.5 \mathrm{~cm} \times$ $5 \mathrm{~cm}$ ) allow for an integrated battery and/or sensor on the top and bottom of the antenna structure. This antenna was fabricated on LCP. The matching networks - double inductive feed and shorting stub, as shown in the figure_can be easily tuned in design to modify the antenna's impedance to match any IC impedance value hence allowing an optimum power transfer from IC to antenna [15]. This is very crucial since most ICs used in passive RFID systems feature a reactive load. It has to be noted that the antenna has to be compact (smaller than $3 \times 3$ in 2 ) with efficiency close to $100 \%$. The tapering of the two arms allows for a higher bandwidth than typical dipole antenna configurations. The simulation and the measurement results are shown in Figure 4, with IC impedance 73-j113 $\Omega$, demonstrating very good agreement and verifying the efficient operation of the antenna in both European and North American bands (860-930 MHz). The bandwidth of operation is defined by the Voltage Standing Wave Ratio (VSWR) of 2 or alternately return loss of $-9.6 \mathrm{~dB}$.

\subsection{Polarization-diversity solutions for harsh environments}

Most antennas in UHF RFID tags, similar to the one shown in Figure 3, are linearly (vertical or horizontal) polarized. In the presence of harsh environments (e.g., containers, industrial machinery) with multiple reflections (i.e., metal) which cause multipath effects, the transmitted/received plane waves undergo polarization changes (depolarization). For instance, a vertically polarized transmitted wave can reach a tag at its blind spot in the radiation pattern. This causes the RFID tag

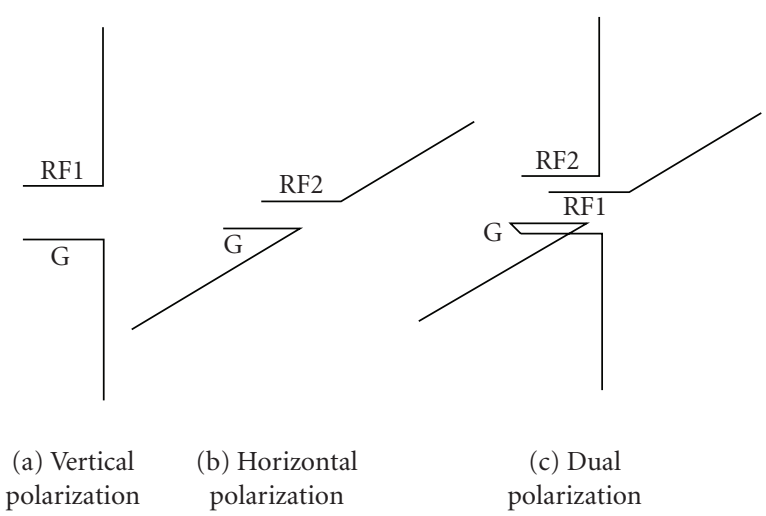

FIgure 5: Polarization definition in dual antenna design.

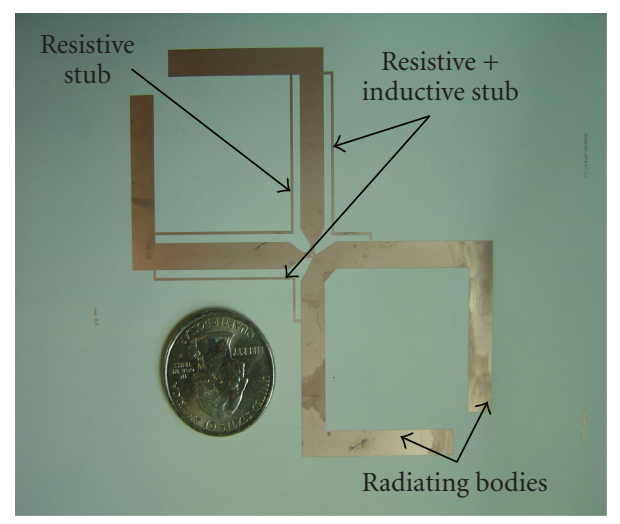

Figure 6: Dual antenna structure.

not to be read. In order to prevent this, in this paper polarization diversity is proposed for a better readability of UHF RFID tags, including the use of both vertical and linear polarized antennas. For dipole antennas, the two orthogonalpolarization antennas can be oriented orthogonal to each other as shown in Figure 5. These two antennas are identical in dimensions and shape, so that the identical signals arriving at these two different branches are in-phase and uncorrelated. The two antennas are also connected with a shorting stub to guarantee an in-phase arrival of both polarization signals to the demodulator in the IC, when the data needs to be retrieved from the combined reception of the two antennas.

Figure 6 shows a $7.5 \mathrm{~cm} \times 5 \mathrm{~cm}$ dual polarized antenna. The shorting stub that connects the "top left" legs of the design both provides the resistive matching and is also used to dc-short the two orthogonal dipole antennas. The dcshorting is also utilized to receive in-phase signals at the two identical input ports. The "bottom right" legs are connected to the ground to achieve the necessary signal, ground excitation. The connecting stubs are used to change the resistance and inductance of the dual antenna structure to conjugately match to the chip impedance. The bandwidth of this antenna is $7.78 \%(70 \mathrm{MHz})$. The radiation efficiency is 93\% since the current flow adds up constructively for the far-field electromagnetic radiation, something that results in an optimized performance in terms of the read range and 


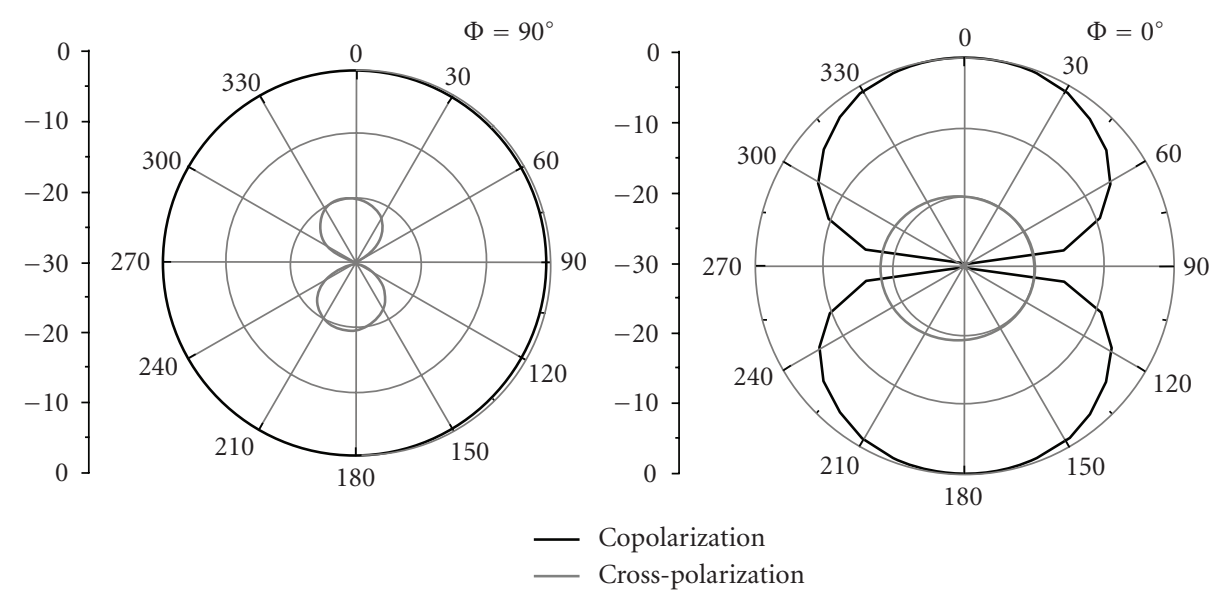

FIGURE 7: Simulated copolarization and cross-polarization radiation patterns.

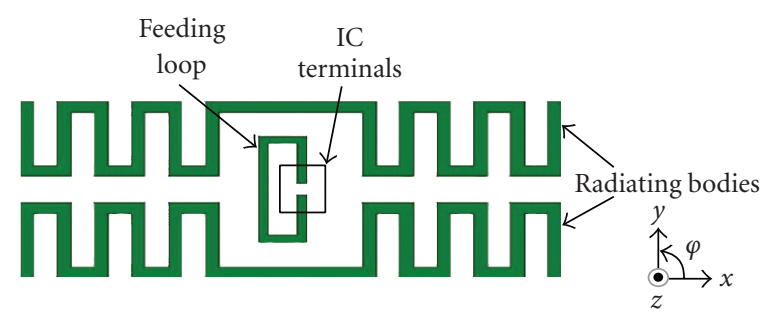

Figure 8: Dual radiating body configuration.

environment versatility. The radiation pattern of the dual polarized antenna is omnidirectional with a maximum directivity of $2.25 \mathrm{~dB}$. Figure 7 shows the copolarization and cross-polarization characteristics of the antenna. A crosspolarization suppression larger than $18 \mathrm{~dB}$ is obtained.

\subsection{Directive antennas for "conveyor-type" applications}

One of the effective methods to increase the read range of the RFID tag to a specific direction besides a good match between the antenna terminals and the IC is a directive radiation pattern. This might be suitable for manufacturing applications, such as boxes, palettes, or items placed on conveyor belts where the position of the tag is known. However, the radiation pattern of most RFID antennas is constrained by their intrinsic dipole nature which is omnidirectional, leaving a limited directivity $(\sim 2 \mathrm{~dB})$. One new topology, named dual-body configuration, is presented in Figure 8. In this figure, two meander-line (radiating structures) arms are placed on each side of the feeding loop as shown. In this case, the current directions are opposite along the arms and the radiation patterns cancel out each other in most of the directions and thus the radiated energy is focused directionally in a dumbbell shape as shown in Figure 9, and a high directivity of $5.60 \mathrm{~dB}$ is observed with $80 \%$ radiation efficiency. In general, a highly increased effective range is expected to achieve with RFID antennas in such a configuration. The dimensions of this dual radiating body antenna are $6.0 \mathrm{~cm} \times 4.20 \mathrm{~cm}$.

\subsection{Inkjet-printed antennas for UHF RFIDs}

Paper-based substrates have a low surface profile depending on appropriate coatings. This is very crucial since fast printing processes, such as direct write methodologies, can be utilized instead of metal etching techniques. A fast process, like inkjet printing, can be used efficiently to print electronics on/in this type of substrates, allowing for a shorter and more reliable fabrication with a resolution of $20 \mu \mathrm{m}$.

The half wavelength tapered width dipole antenna shown in Figure 10 was designed to have a center frequency of $914 \mathrm{MHz}$. Two stubs, inductive and resistive, are used in this antenna similar to Figure 3. The IC used in this design was Philips EPC 1.19 Gen 2 RFID ASIC IC, which exhibits a stable impedance behavior of 16-j350 $\Omega$ over the frequency $902 \mathrm{MHz}-928 \mathrm{MHz}$. The simulated return loss (RL) plot, shown in Figure 11, shows a bandwidth of $905 \mathrm{MHz}-$ $925 \mathrm{MHz}$. The RFID antenna was inkjet-printed with overall dimensions of $8.2 \mathrm{~cm} \times 4.5 \mathrm{~cm}$ featuring a radiation pattern that is quite similar to that of a classic dipole as shown in Figure 12.

\section{RFID/SENSOR MODULE INTEGRATION}

In order to meet the demand for ultra-low-cost passive, semipassive, and active RFID tags, a simple manufacturing process, such as conductive ink printing technology on organic substrates, is proposed. The ultimate goal is to have an all printed RFID tag (antenna, IC, battery, and sensor) on a lowcost environmental friendly paper. The proposed module for such an RFID/sensor integrated tag is shown in Figures 13 and 14, and is currently under development. The proposed RFID tag will be powered by a lithium ion battery. The battery will be charged by the power induced across the antenna terminals, which will be used to power the IC. The IC will comprise of an RFID communication module, and an interface to an external sensor. The IC interface will be used to sample the analog output of the sensor and perform an analog-to-digital conversion on the recorded sensor output. The digital version of the sensor output will be encoded in the appropriate RFID data scheme using GEN2 protocol, and 


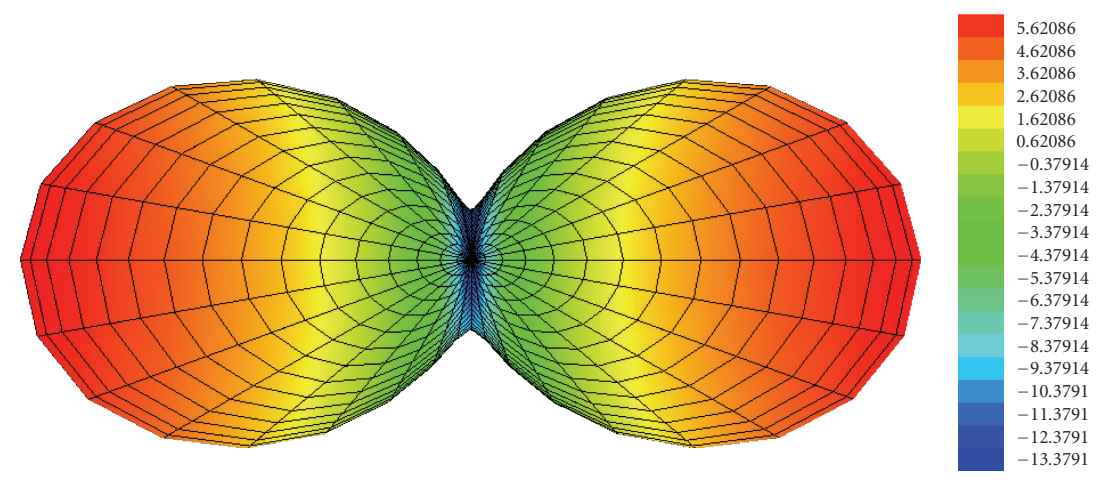

FIGURE 9: Dual radiating body radiation pattern.

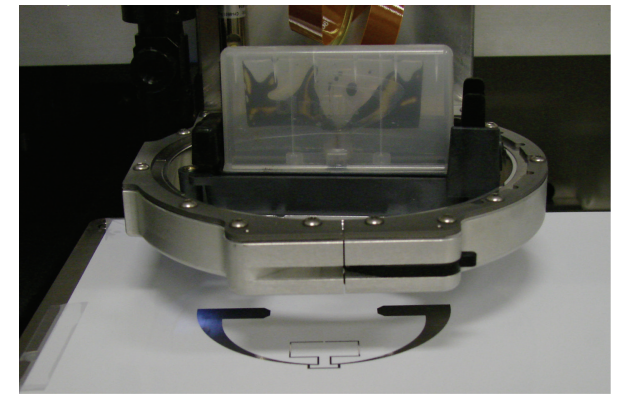

FIGURE 10: Inkjet printed RFID antenna.

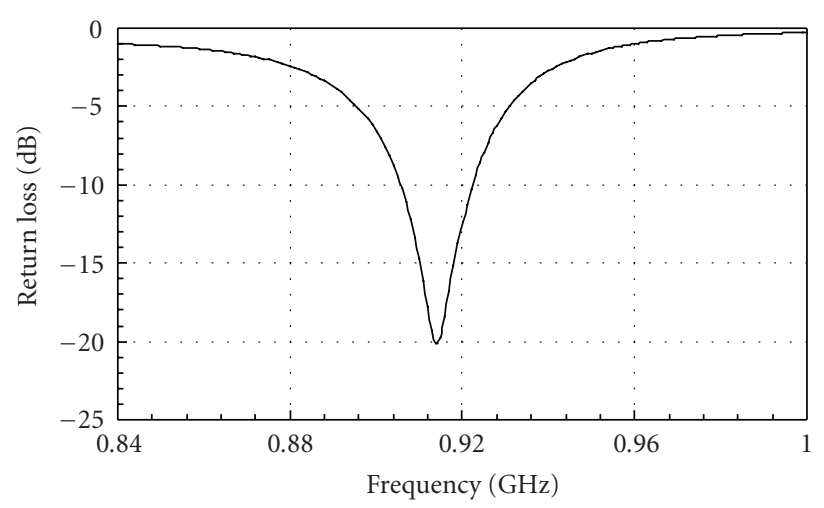

FIGURE 11: Simulated return loss.

will be transmitted to the RF communication module of the IC. The RFID communication module will be used to transmit this sensor data to the reader.

Integration of the IC to the tag will require fabrication techniques that can withstand the rigors of packaging while at the same time provide electrically good contact between the IC and the antenna. In addition, operation in the UHF range also requires the packaging to introduce as minimal parasitic effects as possible to ensure good impedance match between the antenna and the IC. One of the ways considered of integrating the ICs is using flip-chip technology [16]. This method does not require any wire bonds but uses solder bumps mounted on the IC chip pads. The chip is then

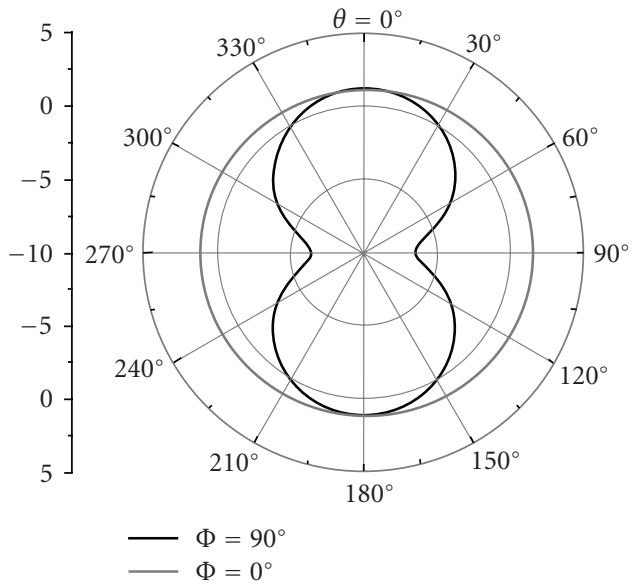

FIgURE 12: Simulated radiation pattern.

mounted upside down in/on the package and the solder reflowed under high temperature. Flip-chip assembly provides an advantage in size, cost, and performance and introduces lower parasitic compared to DIP and SOP packaging. Wire bonding [17] is another common method used to make interconnections between the IC and the antenna terminals. This method involves the use of very thin wires (typically $\sim 15 \mu \mathrm{m}$ ) made of gold or aluminum to make interconnects. Wire bonding is generally considered the most cost-effective and flexible interconnect technology, and is used to assemble the vast majority of semiconductor packages. Many of the ICs also still come in Thin Shink Small Outline Package (TSSOP) [18] that needs to be soldered onto the circuit. The main disadvantage with TSSOP packaging is that they introduce more parasitic effects into the circuit compared to flip-chip and wire bonding technology.

Battery is another crucial part in the RFID/sensor module. One of the major concerns is the limited lifetime of the batteries. The cost of replacing batteries can be relatively high. The same issue is of major importance in active and semipassive RFID tags. Three different power source types are available at the moment: power reservoirs, power distribution methods, and power scavenging sources. 


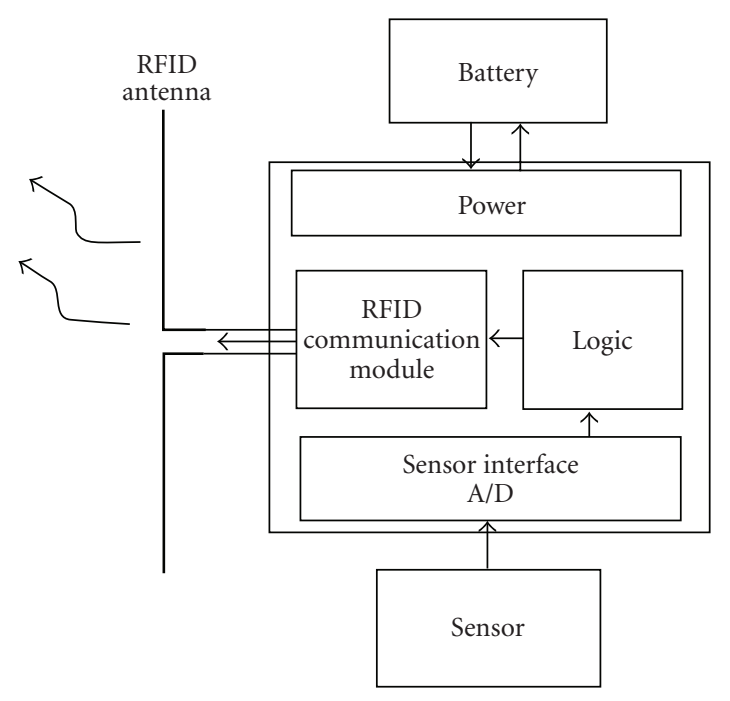

FiguRE 13: Diagram of RFID/sensors tag.

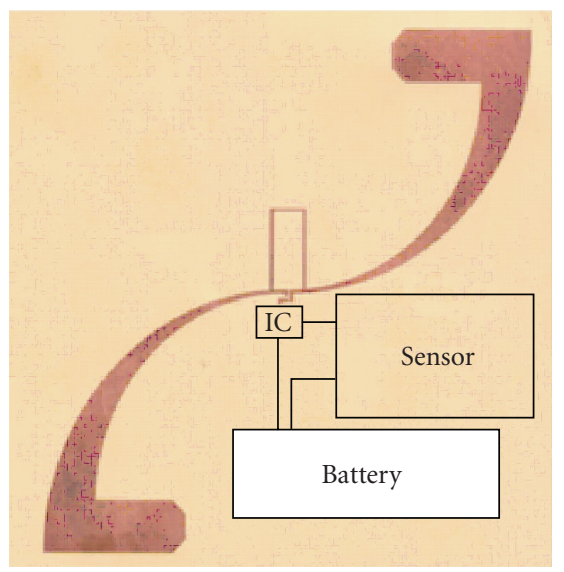

FIgURE 14: Suggested outline of integrated S-shaped antenna with IC, sensor, and embedded battery.

Among the power reservoir technologies investigated over the past years [19], rechargeable lithium thin film batteries seem to be the most suitable solution to be embedded in organic substrates due to their small thickness. The active RFID tag being currently developed will be powered by a lithium ion battery that has been developed and is $300 \mu \mathrm{m}$ thick. The battery will be charged by the power induced across the antenna terminals through a continuous mode AC-DC power converter. Such batteries have rechargeable capabilities which overcome short lifetime limitations, thus making them extremely useful for the drive of the sensors in active and semipassive RFID tags. Active tags solely utilize the battery to power the sensor and the IC, the semipassive need a power distribution method (i.e., electromagnetic power transmission) to operate the IC, thus allowing the sensor to use the battery as an independent power source. The main advantage of the reservoir source is that it eliminates the need for the label to collect energy from the reader, permitting the transmission of relatively large amounts of data over long distances ( $>100 \mathrm{ft})$, while improving its signal-tonoise ratio. On the other hand, electromagnetic power transmission used with a battery allows for a reduced battery consumption and an increased the lifetime. Among the power scavenging sources, thin film solar arrays play a major advantage in outdoor applications with difficult access and impossibility of using wires. Power scavenging may use pressure as a mean of energy conversion by using a piezoelectric printed collector [20]. Solar cells may use light as an efficient way; where recent developments have used printed solar cells for this purpose [21]. Temperature from environmental surroundings such as human body heat in wearable electronics utilizing a modified Carnot cycle (with a projected efficiency of 50\%) is also one of the good candidates [22].

\section{CONCLUSIONS}

In this paper, an overview of novel design and integration approaches for improved performance "enhancedcognition" UHF passive and active radio frequency identification (RFID) tags is reported. Antenna design rules are explained for a variety of applications while addressing a variety of practical UHF RFID issues (worldwide frequency coverage, harsh environments, max-range enhanceddirectivity). Embedded power sources and integration of sensor and integrated circuits (ICs) are also conceptually investigated on low-cost organic substrates, such as liquid crystal polymer (LCP) and paper, enabling the use of inkjetprinting capability for the UHF frequency band. The proposed technologies could potentially enable the low-cost implementation of large-scale ad hoc networks that could potentially offer the capabilities of "ubiquitous sensing" and "cognitive intelligence" for a variety of security, military, logistics, automotive, and pharmaceutical applications.

\section{REFERENCES}

[1] K. Finkenzeller, RFID Handbook: Fundamentals and Applications in Contactless Smart Cards and Identification, John Wiley \& Sons, New York, NY, USA, 2nd edition, 2004.

[2] R. Weinstein, "RFID: a technical overview and its application to the enterprise," IT Professional, vol. 7, no. 3, pp. 27-33, 2005.

[3] T. Carrico and F. Perich, "Cognitive situation monitoring and awareness of grid systems," in Proceedings of IEEE Military Communications Conference (MILCOM '05), vol. 3, pp. 18071812, Atlatnic City, NJ, USA, October 2005.

[4] A. Ferrer-Vidal, A. Rida, S. Basat, L. Yang, and M. M. Tentzeris, "Integration of sensors and RFID's on ultra-low-cost paperbased substrates for wireless sensor networks applications," in Proceedings of the 2nd IEEE Workshop on Wireless Mesh Networks (WiMesh '06), pp. 126-128, Reston, Va, USA, September 2006.

[5] V. Subramanian, J. M. J. Fréchet, P. C. Chang, et al., "Progress toward development of all-printed RFID tags: materials, processes, and devices," Proceedings of the IEEE, vol. 93, no. 7, pp. 1330-1338, 2005.

[6] B. Farrell and M. St. Lawrence, "The processing of liquid crystalline polymer printed circuits," in Proceedings of the 52nd Electronic Components and Technology Conference (ECTC '02), pp. 667-671, San Diego, Calif, USA, May 2002. 
[7] K. Jayaraj, T. E. Noll, and D. Singh, "RF characterization of a low cost multichip packaging technology for monolithic microwave and millimeter wave integrated circuits," in Proceedings of the 3rd International Symposium on Signals, Systems and Electronics (URSI'95), pp. 443-446, October 1995.

[8] G. Zou, H. Gronqvist, P. Starski, and J. Liu, "High frequency characteristics of liquid crystal polymer for systemin a package application," in Proceedings of the 8th International Symposium on Advanced Packaging Materials (ISAPM '02), pp. 337-341, Stone Mountain, Ga, USA, March 2002.

[9] G. Zou, H. Grönqvist, J. P. Starski, and J. Liu, "Characterization of liquid crystal polymer for high frequency system-in-apackage applications," IEEE Transactions on Advanced Packaging, vol. 25, no. 4, pp. 503-508, 2002.

[10] A. Piqué and D. B. Chrisey, Eds., Direct-Write Technologies for Rapid Prototyping Applications, Academic Press, San Diego, Calif, USA, 2002.

[11] M. C. Lessard, L. van Nifterik, M. Masse, J. F. Penneau, and R. Grob, "Thermal aging study of insulating papers used in power transformers," in Proceedings of the Annual Report of the Conference on Electrical Insulation and Dielectric Phenomena (CEIDP '96), vol. 2, pp. 854-859, Millbrae, Calif, USA, October 1996.

[12] S. Simula, S. Ikäläinen, K. Niskanen, T. Varpula, H. Seppä, and A. Paukku, "Measurement of the dielectric properties of paper," Journal of Imaging Science and Technology, vol. 43, no. 5, pp. 472-477, 1999.

[13] D. C. Thompson, O. Tantot, H. Jallageas, G. E. Ponchak, M. M. Tentzeris, and J. Papapolymerou, "Characterization of liquid crystal polymer (LCP) material and transmission lines on LCP substrates from 30 to $110 \mathrm{GHz}$," IEEE Transactions on Microwave Theory and Techniques, vol. 52, no. 4, pp. 1343-1352, 2004.

[14] L. Yang, S. Basat, A. Rida, and M. M. Tentzeris, "Design and development of novel miniaturized UHF RFID tags on ultralow-cost paper-based substrates," in Proceedings of Asia Pacific Microwave Conference (APMC'06), Yokohama, Japan, December 2006.

[15] L. Yang, S. Basat, and M. M. Tentzeris, "Design and development of novel inductively coupled RFID antennas," in Proceedings of IEEE Antennas and Propagation Society International Symposium (APS '06), pp. 1035-1038, Albuquerque, NM, USA, July 2006.

[16] T. Braun, K.-F. Becker, M. Koch, V. Bader, R. Aschenbrenner, and H. Reichl, "Flip chip molding-recent progress in flip chip encapsulation," in Proceedings of the 8th International Symposium on Advanced Packaging Materials (ISAPM '02), pp. 151159, Stone Mountain, Ga, USA, March 2002.

[17] S. L. Khoury, D. J. Burkhard, D. P. Galloway, and T. A. Scharr, "A comparison of copper and gold wire bonding on integrated circuit devices," in Proceedings of the 40th Electronic Components and Technology Conference (ECTC '90), vol. 1, pp. 768776, Las Vegas, Nev, USA, May 1990.

[18] X. J. Zhao and J. F. J. M. Caers, "To simulate the formation of TSSOP solder joint with SAC solder and assess on the effects of the stencil design and the misalignment on the joint shape," in Proceedings of the 6th Electronics Packaging Technology Conference (EPTC '04), pp. 313-317, Singapore, December 2004.

[19] J. B. Bates, N. J. Dudney, B. J. Neudecker, and B. Wang, “Thinfilm lithium batteries," in New Trends in Electrochemical Technology: Energy Storage Systems in Electronics, pp. 453-485, Gordon and Breach, Singapore, 2000.
[20] R. Supino and J. Talghader, "Optical power-scavenging system for fluidic microsensors," in Proceesings of IEEE/LEOS International Conference on Optical MEMS and Their Applications (OMEMS '05), pp. 79-80, Oulu, Finland, August 2005.

[21] K.-Y. Lin, T. K. K. Tsang, M. Sawan, and M. N. El-Gamal, "Radio-triggered solar and RF power scavenging and management for ultra low power wireless medical applications," in Proceedings of IEEE International Symposium on Circuits and Systems (ISCAS '06), pp. 5728-5731, Kos, Greece, May 2006.

[22] R. W. Hart, H. S. White, B. Dunn, and D. R. Rolison, "3-D microbatteries," Electrochemistry Communications, vol. 5, no. 2, pp. 120-123, 2003.

\section{AUTHOR CONTACT INFORMATION}

Li Yang: School of Electrical and Computer Engineering, Georgia Institute of Technology, Atlanta, GA 30332, USA; liyang@ece.gatech.edu

Amin Rida: School of Electrical and Computer Engineering, Georgia Institute of Technology, Atlanta, GA 30332, USA; arida@ece.gatech.edu

Rushi Vyas: School of Electrical and Computer Engineering, Georgia Institute of Technology, Atlanta, GA 30332, USA; gtg904e@mail.gatech.edu

Manos M. Tentzeris: School of Electrical and Computer Engineering, Georgia Institute of Technology, Atlanta, GA 30332, USA; etentze@ece.gatech.edu 

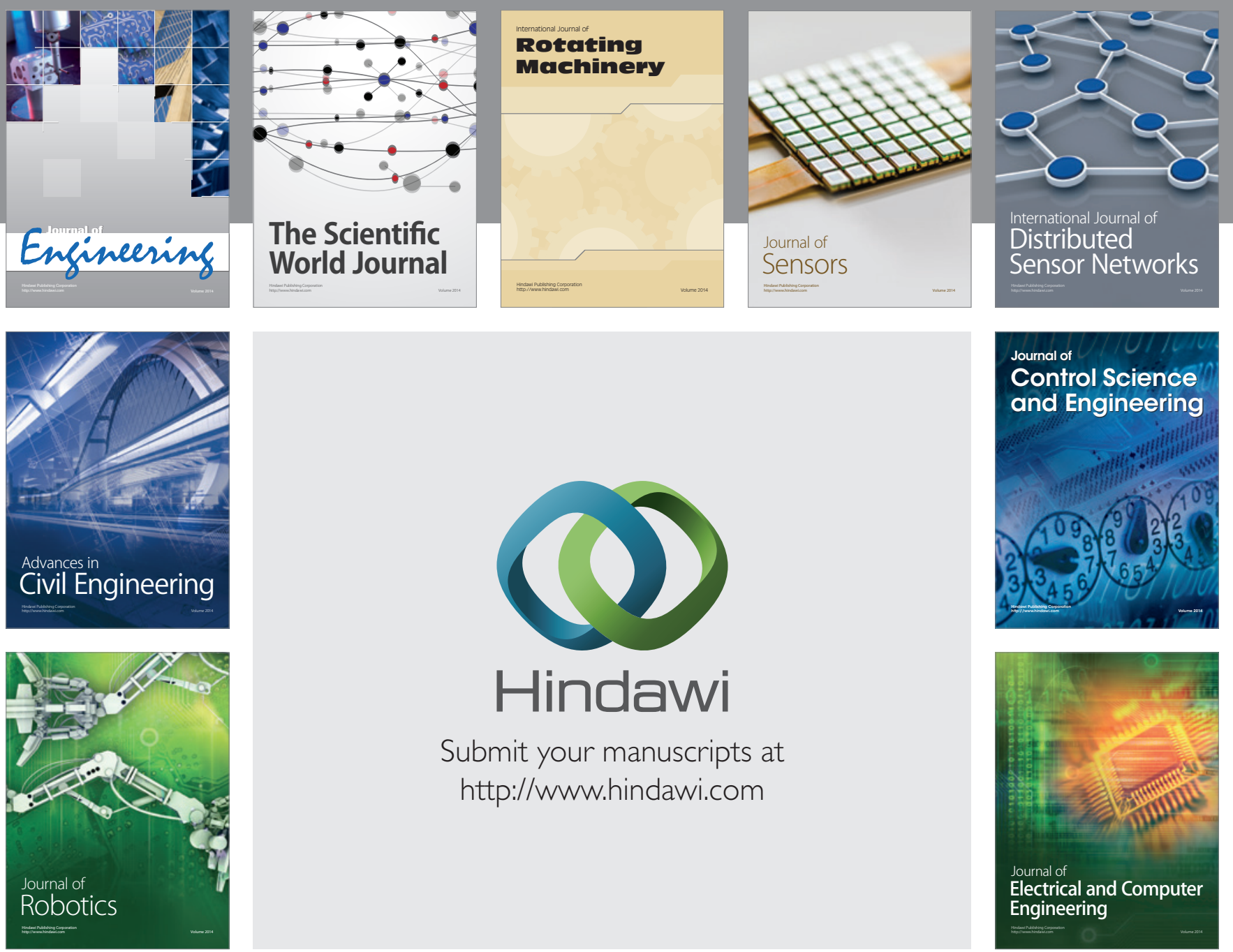

Submit your manuscripts at

http://www.hindawi.com
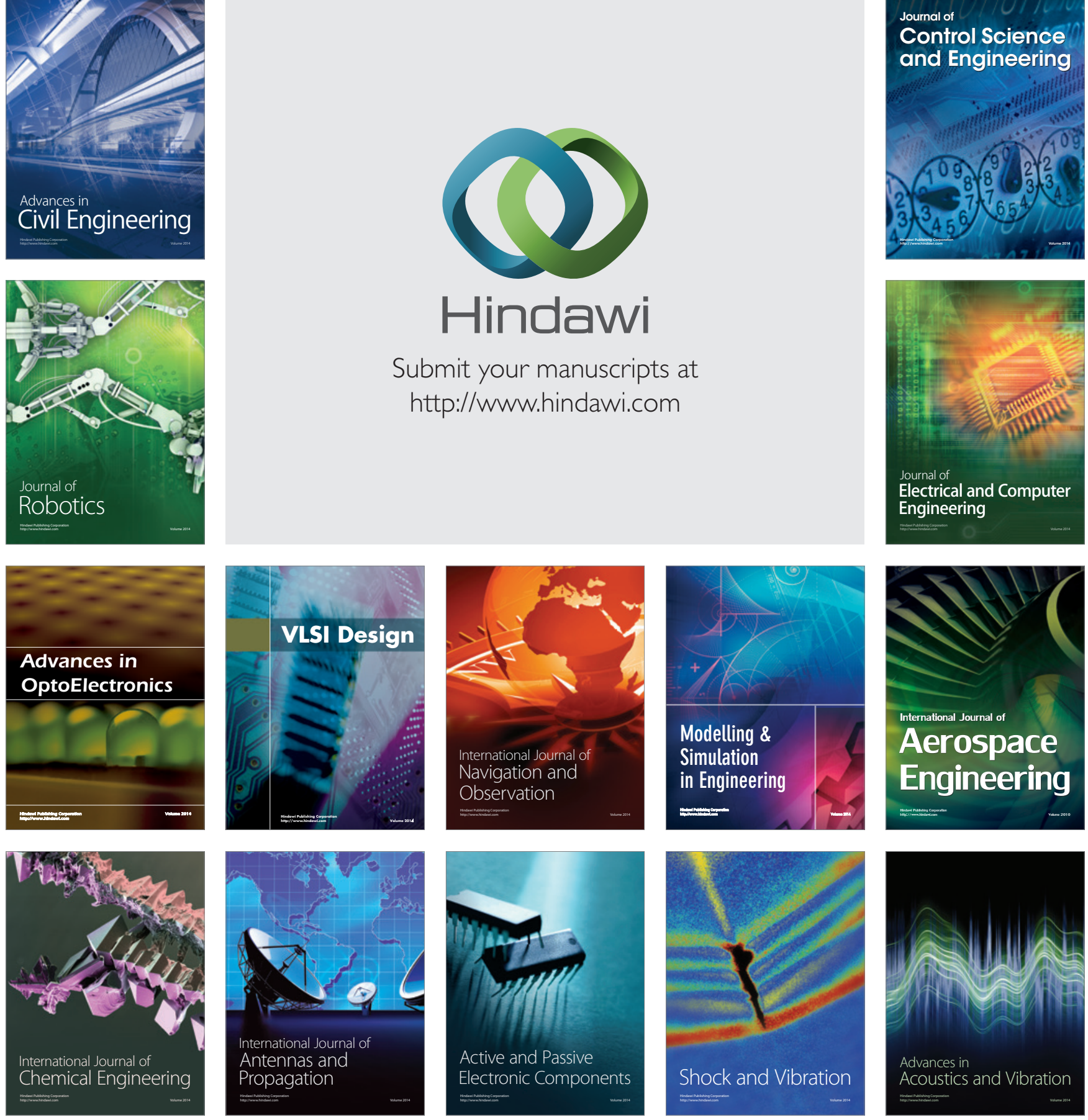